\title{
Physico-chemical characteristics and heavy metal contents in water of Wadi El-Rayan Lakes, western desert, Egypt.
}

\author{
Sayed A. Shama ${ }^{1}$; Mohamed E. Goher ${ }^{2}$; Mohamed H. Abdo ${ }^{2}$; \\ Samy M. Kaial ${ }^{3}$ and Ayman A. Ahmed ${ }^{3}$ \\ 1- Faculty of Science, Benha University, Benha, Egypt \\ 2- National Institutes of Oceanography and Fisheries (NIOF), Cairo, Egypt \\ 3- MTI (Modern university for technology and information), Cairo, Egypt.
}

\begin{abstract}
Dhysico-chemical characteristics and eight heavy metals ( $\mathrm{Fe}, \mathrm{Mn}, \mathrm{Cu}, \mathrm{Co}, \mathrm{Cr}$, $\mathrm{Ni}, \mathrm{Pb}$ and $\mathrm{Cd}$ ) of Wadi El Rayan Lakes water were evaluated from summer 2009 to spring 2010. Salinity (TDS) and major ions show highly significant difference between the two lakes. On the other side, station 1 in $1^{\text {st }}$ lake (infront El Wadi Drain) exhibits the highest content of nutrient salts and heavy metals. The mean values of $\mathrm{Fe}, \mathrm{Mn}, \mathrm{Cu}, \mathrm{Co}, \mathrm{Cr}, \mathrm{Ni}, \mathrm{Pb}$ and $\mathrm{Cd}$ in the $1^{\text {st }}$ lake were $562.33,82.71,15.49,9.69,11.86,11.68,9.66$ and $<0.20 \mu \mathrm{g} / 1$ respectively, on the other hand, the values were $372.00,56.70,10.37,7.72,9.72,9.43,6.79$ and $<0.20 \mu \mathrm{g} / \mathrm{l}$ in the $2^{\text {nd }}$ lake respectively. The present results revealed that the $2^{\text {nd }}$ lake undergoes progressive increase in salinity, especially in the last decade and the lake water may be salinized with time, leading to a great revolutionize in fauna, flora and environment of these lakes. On the other hand, the discharge of pollutants via El Wadi Drain causes unfavorable impact on the lakes environment.
\end{abstract}

Keywords: Wadi El-Rayan Lakes, nutrient salts, heavy metals and water quality.

\section{INTRODUCTION}

A suitable environment is necessary for any organism, since life depends upon the continuance of a proper exchange of essential substances and energies between the organism and its surroundings (Welch, 1952). Based on the discoveries of our satellites, it appears that water is a unique substance in our discovered universe. The presence of water on earth is in itself unique, for the planet earth has few natural liquids (Lawson, 2011). Water is an elixir of life, the $2 / 3$ rd mass of our body is water and $70 \%$ surface of the earth is covered by water (Khare and Jadhav, 2008). It governs the evolution and function of universe on the earth hence water is mother of all living world (Chaurasia and Pandey, 2007). Water is fast becoming a scare commodity in most part of the world (Usharani et al., 2010).

In recent years, more and more attention has been paid to surface water quality. It is difficult to understand the biological phenomenon fully, without the knowledge of water quality, because the chemistry of water reveals much about 
the metabolism of the ecosystem and explains the general hydro biological interrelationship (Sithik et al., 2009).

In the seventies two lakes were created in the lower portion of Wadi El Rayan sub-depression to channel out excess agricultural drainage water in order to slow-down the increase of the water-table in the Fayoum main depression and in the Qarun lake. The creation of a large body of water in this hyper-arid area had a striking ecological impact: new species of plants, mammals, birds and invertebrates moved to Wadi El Rayan area (IUCN, 2000a). These two successive lakes, separated by a waterfall, were created as a reservoir for drainage wastewater (El-Shabrawy and Dumont, 2009). The flow of the drainage water to the northern $1^{\text {st }}$ lake began in April 1973. In 1983 the water flowed through an open canal about $5 \mathrm{~km}$ long and a vertical drop of $2.5 \mathrm{~m}$ over the falls located in the southern $2^{\text {nd }}$ lake. Wadi EI-Rayan lakes receive the agricultural wastewater drainage from El-Wadi Drain and vary in their physical and chemical characters (Ali et al., 2007), which controlling its area and volume (Mohamed and Gad, 2008).

Now, the main threat to the effective long-term protection of the Wadi El-Rayan protected area is seen to be the development of un-controlled economic activities within its boundaries. These activities include large-scale land reclamation schemes, major oil extraction operations, rapidly expanding aquaculture, commercial fishing and tourism, as well as human settlement in highly sensitive areas such as previously un-disturbed habitats used by gazelles and other key species (IUCN, 1998a \& b). furthermore, The extremely high rate of evaporation coupled with very low precipitation, both characteristic of arid areas, and the fact that the lakes are being formed from agricultural drainage wastewater could lead to increased concentration of pollutants and a rapid increase in salinity of the lakes (Saleh et al., 1988), where pollutants from agricultural waste including pesticides and fertilizers as well as other effluents of industrial activities and runoffs certainly will pass into the biotic elements of the ecosystem (Sayed and Abdel-Satar, 2009).

Wadi EI-Rayan lakes are very important economically in fishing, husbandry, ecological tourism and educational and scientific researches. Therefore, the accurate determinations of physical and chemical parameters and heavy metals in aquatic environment are of ultimate important for controlling their pollution. This study aims to provid additional information to existing data on water quality assessments of this important water body besides to the levels of some heavy metals. 


\section{Site Description}

\section{MATERIALS AND METHODS}

Wadi El-Rayan Depression has an area of $203 \mathrm{~km}^{2}$ with two successive lakes connected through a channel (Fouda and Saleh, 1987). The total area of the two lakes is about $113.85 \mathrm{~km}^{2}$ represented as:

The $1^{\text {st }}$ lake: Total area is $55.9 \mathrm{~km}^{2}$, with a total perimeter $47.28 \mathrm{~km}$, the maximum width: $10.37 \mathrm{~km}$ and the maximum length $6.610 \mathrm{~km}$, the maximum depths of the first lake is $23 \mathrm{~m}$, this lake takes a circular shape (EL Bayomi, 2006), and receives frequent effluent of wastewater from El-Wadi Drain (about 200million cubic meters of agricultural drainage water are transported annually) (El-Shabrawy, 2007).

The $2^{\text {nd }}$ lake: Total Area is $57.95 \mathrm{~km}^{2}$, total perimeter $63.81 \mathrm{~km}$ the maximum width: $4.498 \mathrm{~km}$, the maximum length $15.72 \mathrm{~km}$, the maximum depths of the second lake is $33 \mathrm{~m}$, this lake takes a conical shape (EL Bayomi, 2006). It is changing all the time, where newly flooded areas are continuously added at the southwestern side of the lake. The inflow water to the second lake varied from $3.66 \times 10^{6} \mathrm{~m}^{3}$ in July to $17.68 \times 10^{6} \mathrm{~m}^{3}$ in March with a total annual of $127.2 \times$ $10^{6} \mathrm{~m}^{3} /$ year (Abd-Ellah, 1999).

The connecting channel: about $5 \mathrm{Km}$ long and vertical drop of $2.5 \mathrm{~m}$ is characterized by permanent shallow water providing suitable habitat for a continuous cover by emergent aquatic macrophytes thus leading to a swamp formation (EL Bayomi, 2006).

\section{Sampling collection}

The sub-surface water samples were collected seasonally from Wadi ElRayan Lakes at six sites in the period from summer (2009) to spring (2010). Six sites $(1,2 \& 3)$ and $(4,5 \& 6)$ were chosen to represent the $1^{\text {st }}$ and $2^{\text {nd }}$ lake, respectively (Fig. 1). The water samples were collected by a polyvinyl chloride Van Dorn water sampler and kept in ice on the spot. Beside, additional surface water sample from El-Wadi Drain was collected to study its impacts on the lakes environment.

\section{Field Measurements}

The electrical conductivity of the water samples $\left(\mathrm{mS} \mathrm{cm} \mathrm{cm}^{-1}\right)$ was measured by using a conductivity meter (S.C.T.33 YSI), pH by Orion Research Ion Analyzer 399A pH meter and water temperature by an ordinary thermometer. $\mathrm{CO}_{3}{ }^{2-}$ and $\mathrm{HCO}_{3}{ }^{-}$were measured titrimetrically on the spot, where the samples were titrated against standard $\mathrm{H}_{2} \mathrm{SO}_{4}(0.02 \mathrm{~N})$ using phenolphthalein and methyl orange indicators. Dissolved Oxygen (DO) content was determined by azide modification method as specified in American Public Health Association (APHA) (2005). 


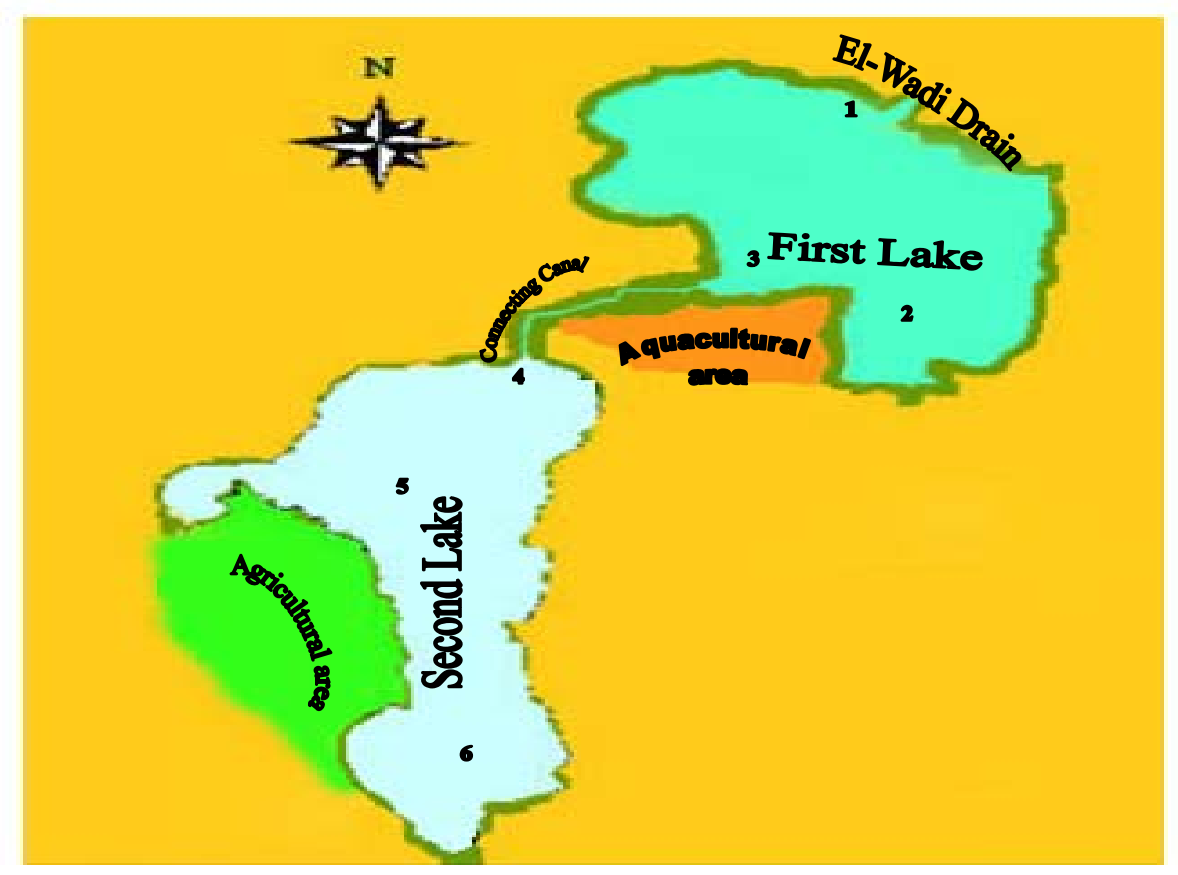

Fig. 1: Map of Wadi El-Rayan Lakes showing the selected stations

\section{Methods of Analysis}

Water samples were analyzed for all selected variables, according to procedures specified in APHA (2005). Total Solids (TS) were measured by evaporating a known volume of well mixed sample, TDS were determined by filtrating a volume of sample with glass micro fiber filter $(\mathrm{GF} / \mathrm{C})$ and a known volume of filtrate was evaporated at $105^{\circ} \mathrm{C}$. Chemical Oxygen Demand (COD) was performed by potassium dichromate oxidation and Biochemical Oxygen Demand (BOD) by 5 days incubation methods. Chloride was determined by argentometric titrimetric method and sulphate by gravimetric methods. Calcium and magnesium were determined by EDTA titrimetric method. Concentrations of nitrite, nitrate, ammonia, orthophosphate (ortho-P) and reactive silicate in water were determined using the calorimetric techniques with formation of reddish purple azo-dye, $\mathrm{Cd}$ reduction, phenate, stannous chloride reduction and molybdosilicate methods, respectively. Total phosphorus (T P) was measured as reactive phosphate after persulphate digestion. Total $\mathrm{Fe}, \mathrm{Mn}, \mathrm{Cu}, \mathrm{Co}, \mathrm{Pb}, \mathrm{Ni}, \mathrm{Cr}$ and $\mathrm{Cd}$ in water were measured after digestion using an atomic absorption spectrophotometer (Perkin Elmer 3110 USA) with graphite atomizer HGA-600.

\section{Statistical Analysis of the Data}

Statistical analysis (standard error "SE") was carried out according to Fisher (1970). LSD (Least significant difference) test was used to compare the significant differences between means of treatment (Waller and Duncan, 1969). 
The statistical package for social science S.P.S.S. (1999) program version 10 was used for all analysis.

\section{RESULTS}

The mean and Standard Error (SE) of the studied physicochemical Characteristics and heavy metals in water samples are presented in Tables (1\&2).

Table 1: Seasonal variations of physico-chemical parameters and heavy metals in water of the $1^{\text {st }}$ Lake.

\begin{tabular}{|c|c|c|c|c|}
\hline Variable & Summer & Autumn & Winter & Spring \\
\hline Temperature $\left({ }^{\circ} \mathrm{C}\right)$ & ${ }^{\mathrm{A}} 30.13^{\mathrm{a}} \pm 0.59$ & ${ }^{\mathrm{A}} 20.70^{\mathrm{c}} \pm 0.06$ & ${ }^{\mathrm{A}} 18.00^{\mathrm{d}} \pm 0.10$ & ${ }^{\mathrm{A}} 23.87^{\mathrm{b}} \pm 0.22$ \\
\hline $\mathrm{EC}(\mathrm{mS} / \mathrm{cm})$ & ${ }^{\mathrm{B}} 2.92^{\mathrm{a}} \pm 0.05$ & ${ }^{\mathrm{B}} 2.74^{\mathrm{b}} \pm 0.05$ & ${ }^{\mathrm{B}} 2.68^{\mathrm{b}} \pm 0.04$ & ${ }^{\mathrm{B}} 2.78^{\mathrm{ab}} \pm 0.06$ \\
\hline $\mathrm{TS}(\mathrm{mg} / \mathrm{l})$ & ${ }^{\mathrm{B}} 1836.00^{\mathrm{a}} \pm 31.64$ & ${ }^{\mathrm{B}} 1760.00^{\mathrm{ab}} \pm 23.12$ & ${ }^{\mathrm{B}} 1733.67^{\mathrm{b}} \pm 20.27$ & ${ }^{\mathrm{B}} 1789.33^{\mathrm{ab}} \pm 26.60$ \\
\hline TDS (mg/l) & ${ }^{\mathrm{B}} 1759.67^{\mathrm{a}} \pm 28.15$ & ${ }^{\mathrm{B}} 1645.67^{\mathrm{b}} \pm 31.65$ & ${ }^{\mathrm{B}} 1617.00^{\mathrm{b}} \pm 25.70$ & ${ }^{\mathrm{B}} 1672.00^{\mathrm{ab}} \pm 33.38$ \\
\hline $\mathrm{TSS}(\mathrm{mg} / \mathrm{l})$ & ${ }^{\mathrm{B}} 76.33^{\mathrm{b}} \pm 12.90$ & ${ }^{\mathrm{A}} 114.33^{\mathrm{a}} \pm 14.44$ & ${ }^{\mathrm{B}} 116.67^{\mathrm{a}} \pm 9.56$ & ${ }^{\mathrm{B}} 117.33^{\mathrm{a}} \pm 8.44$ \\
\hline $\mathrm{pH}$ & ${ }^{\mathrm{A}} 8.60^{\mathrm{b}} \pm 0.06$ & ${ }^{\mathrm{A}} 8.75^{\mathrm{ab}} \pm 0.01$ & ${ }^{\mathrm{A}} 8.59^{\mathrm{b}} \pm 0.06$ & ${ }^{\mathrm{A}} 8.90^{\mathrm{a}} \pm 0.04$ \\
\hline $\mathrm{DO}(\mathrm{mg} / \mathrm{l})$ & ${ }^{\mathrm{A}} 6.60^{\mathrm{b}} \pm 0.12$ & ${ }^{\mathrm{A}} 9.70^{\mathrm{a}} \pm 0.12$ & ${ }^{\mathrm{A}} 8.27^{\mathrm{ab}} \pm 0.77$ & ${ }^{\mathrm{A}} 10.67^{\mathrm{a}} \pm 0.53$ \\
\hline $\operatorname{BOD}(\mathrm{mg} / \mathrm{l})$ & ${ }^{\mathrm{A}} 6.87^{\mathrm{a}} \pm 0.18$ & ${ }^{\mathrm{A}} 3.56^{\mathrm{b}} \pm 0.42$ & ${ }^{\mathrm{A}} 3.47^{\mathrm{b}} \pm 0.18$ & ${ }^{\mathrm{A}} 6.07^{\mathrm{a}} \pm 0.35$ \\
\hline $\operatorname{COD}(\mathrm{mg} / \mathrm{l})$ & ${ }^{\mathrm{A}} 8.13^{\mathrm{ab}} \pm 1.27$ & ${ }^{\mathrm{B}} 9.87^{\mathrm{a}} \pm 0.20$ & ${ }^{\mathrm{B}} 5.47^{\mathrm{b}} \pm 0.48$ & ${ }^{\mathrm{A}} 9.60^{\mathrm{a}} \pm 1.22$ \\
\hline $\mathrm{NH}_{3}(\mu \mathrm{g} / \mathrm{l})$ & ${ }^{\mathrm{A}} 228.80^{\mathrm{a}} \pm 74.99$ & ${ }^{\mathrm{A}} 154.47^{\mathrm{a}} \pm 40.22$ & ${ }^{\mathrm{A}} 200.30^{\mathrm{a}} \pm 38.65$ & ${ }^{\mathrm{A}} 212.80^{\mathrm{a}} \pm 59.44$ \\
\hline $\mathrm{NO}_{2}^{-}(\mu \mathrm{g} / \mathrm{l})$ & ${ }^{\mathrm{A}} 14.21^{\mathrm{a}} \pm 7.49$ & ${ }^{\mathrm{A}} 11.90^{\mathrm{a}} \pm 9.73$ & ${ }^{\mathrm{A}} 22.79^{\mathrm{a}} \pm 9.02$ & ${ }^{\mathrm{A}} 17.51^{\mathrm{a}} \pm 5.59$ \\
\hline $\mathrm{NO}_{3}^{-}(\mu \mathrm{g} / \mathrm{l})$ & ${ }^{\mathrm{A}} 117.96^{\mathrm{a}} \pm 51.43$ & ${ }^{\mathrm{A}} 139.36^{\mathrm{a}} \pm 65.37$ & ${ }^{\mathrm{A}} 157.87^{\mathrm{a}} \pm 66.24$ & ${ }^{\mathrm{A}} 96.87^{\mathrm{a}} \pm 62.61$ \\
\hline Ortho-P $(\mu \mathrm{g} / \mathrm{l})$ & ${ }^{\mathrm{A}} 57.03^{\mathrm{a}} \pm 7.49$ & ${ }^{\mathrm{A}} 34.93^{\mathrm{a}} \pm 9.73$ & ${ }^{\mathrm{A}} 41.57^{\mathrm{a}} \pm 9.02$ & ${ }^{\mathrm{A}} 43.00^{\mathrm{a}} \pm 5.59$ \\
\hline Total-P $(\mu \mathrm{g} / \mathrm{l})$ & ${ }^{\mathrm{A}} 312.83^{\mathrm{a}} \pm 38.41$ & ${ }^{\mathrm{A}} 302.93^{\mathrm{a}} \pm 65.84$ & ${ }^{\mathrm{A}} 270.40^{\mathrm{a}} \pm 76.60$ & ${ }^{\mathrm{A}} 190.13^{\mathrm{a}} \pm 27.90$ \\
\hline $\mathrm{SiO}_{2}{ }^{2-}(\mathrm{mg} / \mathrm{l})$ & ${ }^{\mathrm{A}} 10.06^{\mathrm{a}} \pm 0.38$ & ${ }^{\mathrm{A}} 7.10^{\mathrm{b}} \pm 0.67$ & ${ }^{\mathrm{B}} 11.71^{\mathrm{a}} \pm 0.67$ & ${ }^{\mathrm{B}} 10.47^{\mathrm{a}} \pm 0.23$ \\
\hline $\mathrm{CO}_{3}{ }^{2-}(\mathrm{mg} / \mathrm{l})$ & ${ }^{\mathrm{A}} 17.33^{\mathrm{a}} \pm 2.67$ & ${ }^{\mathrm{A}} 20.00^{\mathrm{b}} \pm 0.00$ & ${ }^{\mathrm{A}} 16.00^{\mathrm{b}} \pm 2.00$ & ${ }^{\mathrm{A}} 29.33^{\mathrm{b}} \pm 1.33$ \\
\hline $\mathrm{HCO}_{3}^{-}(\mathrm{mg} / \mathrm{l})$ & ${ }^{\mathrm{A}} 243.65^{\mathrm{a}} \pm 3.61$ & ${ }^{\mathrm{B}} 211.63^{\mathrm{b}} \pm 10.38$ & ${ }^{\mathrm{A}} 247.47^{\mathrm{a}} \pm 3.41$ & ${ }^{\mathrm{A}} 252.59^{\mathrm{a}} \pm 6.15$ \\
\hline $\mathrm{Cl}^{-}(\mathrm{mg} / \mathrm{l})$ & ${ }^{\mathrm{B}} 661.67^{\mathrm{a}} \pm 27.12$ & ${ }^{\mathrm{B}} 643.72^{\mathrm{a}} \pm 19.58$ & ${ }^{\mathrm{B}} 597.27^{\mathrm{a}} \pm 18.62$ & ${ }^{\mathrm{A}} 642.60^{\mathrm{a}} \pm 21.04$ \\
\hline $\mathrm{SO}_{4}{ }^{2-}(\mathrm{mg} / \mathrm{l})$ & ${ }^{\mathrm{B}} 350.00^{\mathrm{a}} \pm 11.72$ & ${ }^{\mathrm{B}} 307.20^{\mathrm{a}} \pm 12.66$ & ${ }^{\mathrm{B}} 306.90^{\mathrm{a}} \pm 13.01$ & ${ }^{\mathrm{B}} 336.78^{\mathrm{a}} \pm 13.28$ \\
\hline $\mathrm{Ca}^{2+}(\mathrm{mg} / \mathrm{l})$ & ${ }^{\mathrm{B}} 66.12^{\mathrm{a}} \pm 1.15$ & ${ }^{\mathrm{B}} 61.47^{\mathrm{a}} \pm 1.67$ & ${ }^{\mathrm{B}} 58.97^{\mathrm{a}} \pm 3.95$ & ${ }^{\mathrm{B}} 63.97^{\mathrm{a}} \pm 1.31$ \\
\hline $\mathrm{Mg}^{2+}(\mathrm{mg} / \mathrm{l})$ & ${ }^{\mathrm{B}} 111.73^{\mathrm{a}} \pm 4.28$ & ${ }^{\mathrm{B}} 103.07^{\mathrm{ab}} \pm 2.82$ & ${ }^{\mathrm{B}} 100.28^{\mathrm{b}} \pm 1.68$ & ${ }^{\mathrm{B}} 105.17^{\mathrm{ab}} \pm 2.35$ \\
\hline $\mathrm{Fe}(\mu \mathrm{g} / \mathrm{l})$ & ${ }^{\mathrm{A}} 778.67^{\mathrm{a}} \pm 110.34$ & ${ }^{\mathrm{A}} 594.67^{\mathrm{ab}} \pm 147.86$ & ${ }^{\mathrm{A}} 252.00^{\mathrm{b}} \pm 75.02$ & ${ }^{\mathrm{A}} 624.00^{\mathrm{a}} \pm 86.00$ \\
\hline $\mathrm{Mn}(\mu \mathrm{g} / 1)$ & ${ }^{\mathrm{A}} 95.52^{\mathrm{a}} \pm 13.25$ & ${ }^{\mathrm{A}} 101.33^{\mathrm{a}} \pm 5.46$ & ${ }^{\mathrm{A}} 38.67^{\mathrm{b}} \pm 20.70$ & ${ }^{\mathrm{A}} 95.33^{\mathrm{a}} \pm 5.76$ \\
\hline $\mathrm{Cu}(\mu \mathrm{g} / \mathrm{l})$ & ${ }^{\mathrm{A}} 15.22^{\mathrm{ab}} \pm 1.75$ & ${ }^{\mathrm{A}} 13.43^{\mathrm{b}} \pm 0.90$ & ${ }^{\mathrm{A}} 21.49^{\mathrm{a}} \pm 3.77$ & ${ }^{\mathrm{A}} 11.83^{\mathrm{b}} \pm 0.79$ \\
\hline Co $(\mu \mathrm{g} / 1)$ & ${ }^{\mathrm{A}} 9.96^{\mathrm{ab}} \pm 0.97$ & ${ }^{\mathrm{A}} 7.31^{\mathrm{b}} \pm 0.56$ & ${ }^{\mathrm{A}} 12.71^{\mathrm{a}} \pm 1.81$ & ${ }^{A} 8.78^{b} \pm 0.87$ \\
\hline $\mathrm{Pb}(\mu \mathrm{g} / 1)$ & ${ }^{\mathrm{A}} 13.13^{\mathrm{a}} \pm 1.79$ & ${ }^{\mathrm{A}} 7.53^{\mathrm{b}} \pm 0.91$ & ${ }^{\mathrm{A}} 9.67^{\mathrm{ab}} \pm 1.44$ & ${ }^{\mathrm{A}} 8.32^{\mathrm{b}} \pm 0.54$ \\
\hline $\mathrm{Ni}(\mu \mathrm{g} / 1)$ & ${ }^{\mathrm{A}} 17.99^{\mathrm{a}} \pm 3.79$ & ${ }^{\mathrm{A}} 9.53^{\mathrm{b}} \pm 0.85$ & ${ }^{\mathrm{A}} 9.52^{\mathrm{b}} \pm 0.99$ & ${ }^{\mathrm{A}} 9.69^{\mathrm{b}} \pm 0.76$ \\
\hline $\mathrm{Cr}(\mu \mathrm{g} / 1)$ & ${ }^{\mathrm{A}} 17.07^{\mathrm{a}} \pm 2.80$ & ${ }^{\mathrm{A}} 8.67^{\mathrm{b}} \pm 0.75$ & ${ }^{\mathrm{A}} 6.99^{\mathrm{b}} \pm 0.82$ & ${ }^{\mathrm{A}} 14.72^{\mathrm{a}} \pm 2.14$ \\
\hline $\mathrm{Cd}(\mu \mathrm{g} / \mathrm{l})$ & $<0.20$ & $<0.20$ & $<0.20$ & $<0.20$ \\
\hline
\end{tabular}

Notes: All values in table (1) are Mean \pm Standard Error (SE) of three replicates. The mean values with different small letter (in the same table) within seasons indicate significant differences $(\mathrm{P}<0.05)$.

The mean values with different capital letter (between $1 \& 2$ tables) within two lakes indicate significant differences $(\mathrm{P}<0.05)$.

Temperature: Temperature is one of the most important ecological factors, which control the physiological behaviour and distribution of organisms. Minimum and maximum temperatures recorded ranged from $14.5^{\circ} \mathrm{C}$ (winter) to $31.3^{\circ} \mathrm{C}$ (summer), respectively. There was no significant difference between two lakes. On the other hand, the results show temporal highly significant difference. 
Table 2: Seasonal variations of physico-chemical parameters and heavy metals in water of the $2^{\text {nd }}$ Lake water.

\begin{tabular}{|c|c|c|c|c|}
\hline Variable & Summer & Autumn & Winter & Spring \\
\hline Temperature $(0 \mathrm{C})$ & ${ }^{A} 30.87^{\mathrm{a}} \pm 0.34$ & ${ }^{\mathrm{A}} 20.63^{\mathrm{C}} \pm 0.33$ & ${ }^{A} 17.10^{d} \pm 1.31$ & ${ }^{\mathrm{A}} 23.70^{\mathrm{b}} \pm 0.12$ \\
\hline $\mathrm{EC}(\mathrm{mS} / \mathrm{cm})$ & ${ }^{\mathrm{A}} 23.46^{\mathrm{a}} \pm 1.68$ & $\mathrm{~A}_{22.43^{\mathrm{a}} \pm 1.59}$ & ${ }^{\mathrm{A}} 21.85^{\mathrm{a}} \pm 1.72$ & $\mathrm{~A}_{22.87^{\mathrm{a}} \pm 1.78}$ \\
\hline $\mathrm{TS}(\mathrm{mg} / \mathrm{l})$ & ${ }^{\mathrm{A}} 14161.00^{\mathrm{a}} \pm 977.50$ & ${ }^{\mathrm{A}} 13357.00^{\mathrm{a}} \pm 957.49$ & ${ }^{\mathrm{A}} 13162.33^{\mathrm{a}} \pm 922.04$ & ${ }^{\mathrm{A}} 13797.33^{\mathrm{a}} \pm 952.20$ \\
\hline TDS (mg/l) & ${ }^{\mathrm{A}} 13772.00^{\mathrm{a}} \pm 943.58$ & ${ }^{\mathrm{A}} 13119.67^{\mathrm{a}} \pm 37.71$ & ${ }^{\mathrm{A}} 12936.00^{\mathrm{a}} \pm 940.58$ & ${ }^{\mathrm{A}} 13510.33^{\mathrm{a}}{ }^{\prime} \pm 1001.05$ \\
\hline $\mathrm{TSS}(\mathrm{mg} / \mathrm{l})$ & ${ }^{\mathrm{A}} 392.33^{\mathrm{a}} \pm 25.79$ & ${ }^{A} 237.33^{b} \pm 45.68$ & $\mathrm{~A}_{2} 26.33^{\mathrm{b}} \pm 14.88$ & ${ }^{\mathrm{A}} 287.00^{\mathrm{ab}} \pm 37.34$ \\
\hline $\mathrm{pH}$ & ${ }^{\mathrm{A}} 8.42^{\mathrm{a}} \pm 0.05$ & ${ }^{\mathrm{A}} 8.49^{\mathrm{a}} \pm 0.11$ & ${ }^{\mathrm{A}} 8.38^{\mathrm{a}} \pm 0.06$ & ${ }^{\mathrm{B}} 8.53^{\mathrm{a}} \pm 0.05$ \\
\hline $\mathrm{DO}(\mathrm{mg} / \mathrm{l})$ & ${ }^{\mathrm{A}} 6.33^{\mathrm{b}} \pm 0.79$ & ${ }^{\mathrm{A}} 8.33^{\mathrm{ab}} \pm 0.85$ & ${ }^{\mathrm{A}} 9.93^{\mathrm{a}} \pm 0.93$ & ${ }^{\mathrm{A}} 10.27^{\mathrm{a}} \pm 0.35$ \\
\hline $\mathrm{BOD}(\mathrm{mg} / \mathrm{l})$ & ${ }^{\mathrm{A}} 6.23^{\mathrm{a}} \pm 0.20$ & ${ }^{\mathrm{A}} 3.00^{\mathrm{C}} \pm 0.10$ & ${ }^{\mathrm{A}} 4.27^{\mathrm{b}} \pm 0.24$ & ${ }^{\mathrm{B}} 4.67^{\mathrm{b}} \pm 0.24$ \\
\hline $\mathrm{COD}(\mathrm{mg} / \mathrm{l})$ & ${ }^{\mathrm{A}} 9.33^{\mathrm{a}} \pm 0.83$ & ${ }^{\mathrm{A}} 11.87^{\mathrm{a}} \pm 0.83$ & ${ }^{\mathrm{A}} 10.00^{\mathrm{a}} \pm 0.83$ & ${ }^{\mathrm{A}} 10.00^{\mathrm{a}} \pm 1.29$ \\
\hline $\mathrm{NH}_{3}(\mu \mathrm{g} / \mathrm{l})$ & ${ }^{\mathrm{A}} 144.63^{\mathrm{a}} \pm 2.63$ & ${ }^{\mathrm{A}} 94.73^{\mathrm{a}} \pm 32.33$ & ${ }^{\mathrm{A}} 112.47^{\mathrm{a}} \pm 20.26$ & ${ }^{\mathrm{A}} 106.87^{\mathrm{a}} \pm 4.35$ \\
\hline $\mathrm{NO}_{2}^{-}(\mu \mathrm{g} / \mathrm{l})$ & ${ }^{\mathrm{A}} 6.71^{\mathrm{a}} \pm 0.77$ & ${ }^{\mathrm{A}} 4.17^{\mathrm{a}} \pm 1.58$ & ${ }^{\mathrm{A}} 10.33^{\mathrm{a}} \pm 3.40$ & ${ }^{\mathrm{A}} 10.32^{\mathrm{a}} \pm 1.43$ \\
\hline $\mathrm{NO}_{3}^{-}(\mu \mathrm{g} / \mathrm{l})$ & ${ }^{\mathrm{A}} 83.14^{\mathrm{a}} \pm 6.15$ & ${ }^{\mathrm{A}} 81.84^{4} \pm 14.65$ & ${ }^{\mathrm{A}} 41.52^{\mathrm{a}} \pm 5.36$ & ${ }^{\mathrm{A}} 66.87^{\mathrm{a}} \pm 19.26$ \\
\hline Ortho-P ( $\mu \mathrm{g} / \mathrm{l})$ & ${ }^{\mathrm{A}} 36.53^{\mathrm{ab}} \pm 0.77$ & ${ }^{\mathrm{A}} 19.43^{\mathrm{b}} \pm 1.58$ & ${ }^{A} 23.10^{b} \pm 3.40$ & ${ }^{\mathrm{A}} 54.93^{\mathrm{a}} \pm 1.43$ \\
\hline Total-P $(\mu \mathrm{g} / \mathrm{l})$ & ${ }^{\mathrm{A}} 261.83^{\mathrm{a}} \pm 35.60$ & ${ }^{\mathrm{A}} 266.67^{\mathrm{a}} \pm 46.50$ & ${ }^{\mathrm{A}} 277.20^{\mathrm{a}} \pm 45.65$ & ${ }^{\mathrm{A}} 218.40^{\mathrm{a}} \pm 5.92$ \\
\hline $\mathrm{SiO}_{2}^{2-}(\mathrm{mg} / \mathrm{l})$ & ${ }^{\mathrm{A}} 9.94^{\mathrm{b}} \pm 0.24$ & ${ }^{\mathrm{A}} 7.30^{\circ} \pm 0.38$ & ${ }^{\mathrm{A}} 14.27^{\mathrm{a}} \pm 0.48$ & ${ }^{\mathrm{A}} 14.91^{\mathrm{a}} \pm 1.46$ \\
\hline $\mathrm{CO}_{3}^{2-}(\mathrm{mg} / \mathrm{l})$ & $\mathrm{A} 10.67^{\mathrm{a}} \pm 1.33$ & ${ }^{\mathrm{A}} 12.67^{\mathrm{a}} \pm 2.91$ & $\mathrm{~A} 8.67^{\mathrm{a}} \pm 2.91$ & ${ }^{\mathrm{B}} 13.33^{\mathrm{a}} \pm 1.76$ \\
\hline $\mathrm{HCO}_{3}^{-}(\mathrm{mg} / \mathrm{l})$ & ${ }^{\mathrm{A}} 221.25^{\mathrm{b}} \pm 7.88$ & $\mathrm{~A}_{2} 250.88^{\mathrm{a}} \pm 8.87$ & $\mathrm{~A}_{2} 247.47^{\mathrm{a}} \pm 1.71$ & ${ }^{\mathrm{A}} 259.41^{\mathrm{a}} \pm 3.41$ \\
\hline $\mathrm{Cl}^{-}(\mathrm{mg} / \mathrm{l})$ & ${ }^{\mathrm{A}} 6430.00^{\mathrm{a}} \pm 444.72$ & ${ }^{\mathrm{A}} 5712.21^{\mathrm{a}} \pm 313.37$ & ${ }^{\mathrm{A}} 5698.48^{\mathrm{a}} \pm 320.09$ & ${ }^{\mathrm{A}} 6131.81^{\mathrm{a}} \pm 430.94$ \\
\hline $\mathrm{SO}_{4}{ }^{2-}(\mathrm{mg} / \mathrm{l})$ & ${ }^{A} 2696.11^{\mathrm{a}} \pm 287.72$ & ${ }^{\mathrm{A}} 2887.33^{\mathrm{a}} \pm 356.92$ & ${ }^{\mathrm{A}} 2859.75^{\mathrm{a}} \pm 320.08$ & ${ }^{A} 2326.52^{a} \pm 324.52$ \\
\hline $\mathrm{Ca}^{2+}(\mathrm{mg} / \mathrm{l})$ & ${ }^{\mathrm{A}} 171.41^{\mathrm{a}} \pm 11.14$ & ${ }^{\mathrm{A}} 157.40^{\mathrm{a}} \pm 6.73$ & ${ }^{\mathrm{A}} 156.29^{\mathrm{a}} \pm 7.29$ & ${ }^{\mathrm{A}} 161.62^{\mathrm{a}} \pm 7.93$ \\
\hline $\mathrm{Mg}^{2+}(\mathrm{mg} / \mathrm{l})$ & ${ }^{\mathrm{A}} 531.45^{\mathrm{a}} \pm 47.46$ & ${ }^{\mathrm{A}} 497.31^{\mathrm{a}} \pm 40.62$ & ${ }^{\mathrm{A}} 517.72^{\mathrm{a}} \pm 39.79$ & ${ }^{\mathrm{A}} 523.70^{\mathrm{a}} \pm 41.88$ \\
\hline $\mathrm{Fe}(\mu \mathrm{g} / \mathrm{l})$ & ${ }^{A} 578.00^{\mathrm{a}} \pm 96.01$ & ${ }^{\mathrm{A}} 624.00^{\mathrm{a}} \pm 86.00$ & ${ }^{\mathrm{A}} 179.33^{\mathrm{C}} \pm 16.83$ & ${ }^{\mathrm{A}} 428.67^{\mathrm{ab}} \pm 21.98$ \\
\hline $\operatorname{Mn}(\mu \mathrm{g} / 1)$ & ${ }^{\mathrm{A}} 59.20^{\mathrm{ab}} \pm 12.57$ & ${ }^{\mathrm{B}} 76.33^{\mathrm{a}} \pm 2.19$ & ${ }^{\mathrm{A}} 26.67^{\mathrm{b}} \pm 21.74$ & ${ }^{\mathrm{B}} 64.33^{\mathrm{ab}} \pm 4.25$ \\
\hline $\mathrm{Cu}(\mu \mathrm{g} / \mathrm{l})$ & ${ }^{\mathrm{A}} 10.67^{\mathrm{a}} \pm 1.00$ & ${ }^{\mathrm{B}} 8.94^{\mathrm{a}} \pm 1.00$ & ${ }^{\mathrm{A}} 12.16^{\mathrm{a}} \pm 1.08$ & ${ }^{\mathrm{A}} 9.73^{\mathrm{a}} \pm 1.12$ \\
\hline $\operatorname{Co}(\mu \mathrm{g} / \mathrm{l})$ & ${ }^{\mathrm{A}} 7.41^{\mathrm{ab}} \pm 0.43$ & ${ }^{\mathrm{A}} 5.27^{\mathrm{b}} \pm 0.61$ & ${ }^{\mathrm{A}} 10.77^{\mathrm{a}} \pm 1.84$ & ${ }^{\mathrm{A}} 7.42^{\mathrm{ab}} \pm 0.61$ \\
\hline $\mathrm{Pb}(\mu \mathrm{g} / \mathrm{l})$ & ${ }^{\mathrm{A}} 8.09^{\mathrm{a}} \pm 1.03$ & ${ }^{\mathrm{A}} 5.96^{\mathrm{bc}} \pm 0.27$ & ${ }^{\mathrm{A}} 7.86^{\mathrm{ab}} \pm 0.38$ & ${ }^{\mathrm{B}} 5.24^{\mathrm{C}} \pm 0.33$ \\
\hline $\mathrm{Ni}(\mu \mathrm{g} / \mathrm{l})$ & ${ }^{\mathrm{A}} 14.27^{\mathrm{a}} \pm 1.00$ & ${ }^{\mathrm{B}} 6.72^{\mathrm{b}} \pm 0.52$ & $\mathrm{~A} 8.75^{\mathrm{b}} \pm 1.16$ & ${ }^{\mathrm{A}} 7.98^{\mathrm{b}} \pm 0.17$ \\
\hline $\operatorname{Cr}(\mu \mathrm{g} / 1)$ & ${ }^{\mathrm{A}} 13.30^{\mathrm{a}} \pm 2.24$ & ${ }^{\mathrm{A}} 8.75^{\mathrm{b}} \pm 1.21$ & ${ }^{\mathrm{A}} 7.49^{\mathrm{b}} \pm 0.64$ & ${ }^{\mathrm{A}} 9.33^{\mathrm{ab}} \pm 0.43$ \\
\hline $\mathrm{Cd}(\mu \mathrm{g} / 1)$ & $<0.20$ & $<0.20$ & $<0.20$ & $<0.20$ \\
\hline
\end{tabular}

Notes: All values in table (2) are Mean \pm Standard Error (SE) of three replicates.

The mean values with different small letter (in the same table) within seasons indicate significant differences $(\mathrm{P}<0.05)$.

The mean values with different capital letter (between $1 \& 2$ tables) within two lakes indicate significant differences $(\mathrm{P}<0.05)$.

Electrical conductivity (EC): EC is the capacity of water to conduct electric current and varies both with number and types of ions in the solution. The values of conductivity and TDS are interrelated. EC ranged between $2.63-3.01 \mathrm{~ms} / \mathrm{cm}$ and $2.63-3.01 \mathrm{~ms} / \mathrm{cm}$ in $1^{\text {st }}$ and $2^{\text {nd }}$ lake respectively with a maximum in summer and a minimum in winter. The significant differences between two lakes are highly. In addition, there were sites significant differences in the $2^{\text {nd }}$ lake.

Total solids (TS): TS refer to the sum of suspended and dissolved matter in water or wastewater. It varied in the $1^{\text {st }}$ and $2^{\text {nd }}$ lake between $(1710-1898 \mathrm{mg} / \mathrm{l})$ and $(11325-15340 \mathrm{mg} / 1)$, respectively where highest values in hot period (summer \& spring) and lowest values in cold period (winter \& autumn). The results were highly significant differences between the $1^{\text {st }}$ and $2^{\text {nd }}$ lake.

Total Dissolved Solids (TDS): TDS is the portion of solids that passes through a filter paper of $0.2 \mathrm{~mm}$ or smaller pore size under specified conditions. TDS 
increased in summer, while the lowest values were recorded in winter. It varied between (1586-1811mg/l) and $(11060-14908 \mathrm{mg} / \mathrm{l})$ in the $1^{\text {st }}$ and $2^{\text {nd }}$ lake, respectively. The differences were a significant between two lakes, as well as spatial significant differences in the $2^{\text {nd }}$ lake.

Total Suspended Solids (TSS): TSS is the portion of solids remnant after the filtration includes organic residues (APHA, 2005). It ranged between (62$136 \mathrm{mg} / \mathrm{l})$ and $(146-432 \mathrm{mg} / \mathrm{l})$ in the $1^{\text {st }}$ and $2^{\text {nd }}$ lakes respectively. On the other hand, there is a significant difference between two lakes. On the other hand, the results show temporal significant difference.

The hydrogen ion concentration (pH): $\mathrm{pH}$ of Wadi El Rayan Lakes lies in alkaline side and it ranged from 8.47 in (winter) to 8.96 in (spring) and from 8.26 in (winter) to 8.71 in (autumn) in the $1^{\text {st }}$ and $2^{\text {nd }}$ lakes respectively.

Dissolved oxygen (DO): DO content indicates the health and ability of the waterbody to purify itself through biochemical processes. DO varied between $(6.4-12 \mathrm{mg} / \mathrm{l})$ and $(4.80-11.4 \mathrm{mg} / \mathrm{l})$ in the $1^{\text {st }}$ and $2^{\text {nd }}$ lakes respectively, with seasonally significant difference with no local difference. The lowest value was recorded during summer, while the highest one during autumn.

Biological oxygen demand (BOD): BOD test was found to be more sensitive test for organic pollution. It ranged between $(3.08-7.20 \mathrm{mg} / \mathrm{l})$ and $(2.80-6.54 \mathrm{mg} / \mathrm{l})$ in the $1^{\text {st }}$ and $2^{\text {nd }}$ lakes respectively, where minimum and maximum values recorded in autumn and summer seasons, respectively. On the other hand, there was temporal significant difference.

Chemical oxygen demand (COD): COD ranged from $4.80 \mathrm{mg} / 1$ to $12.00 \mathrm{mg} / \mathrm{l}$ and $8.00 \mathrm{mg} / 1$ to $12.40 \mathrm{mg} / 1$ in the $1^{\text {st }}$ and $2^{\text {nd }}$ lake, respectively with significant difference during the cold seasons (winter and autumn). The highest value was recorded during spring, while the lowest one during winter.

Nutrient Salts: The nutrient salts include compounds that contain nitrogen $\left(\mathrm{NH}_{3}, \mathrm{NO}_{2}^{-}, \mathrm{NO}_{3}{ }^{-}\right)$, phosphours (ortho-P, TP) or silicate $\left(\mathrm{SiO}_{2}{ }^{2-}\right)$ in different forms either in available or non-available forms. The ranges of $\mathrm{NH}_{3}, \mathrm{NO}_{2}{ }^{-}$and $\mathrm{NO}_{3}{ }^{-}$in the $1^{\text {st }}$ lake are $(85.70-376.80 \mu \mathrm{g} / \mathrm{l}),(1.90-40.80 \mu \mathrm{g} / \mathrm{l})$ and $(26.55-290.0$ $\mu \mathrm{g} / 1)$, respectively while the ranges of ortho-P and TP are $(27.50-75.90 \mu \mathrm{g} / \mathrm{l})$, and $(154.80-434.00 \mu \mathrm{g} / 1)$, respectively, with spatial significant difference. In the $2^{\text {nd }}$ lake, the ranges of $\mathrm{NH}_{3}, \mathrm{NO}_{2}{ }^{-}$and $\mathrm{NO}_{3}{ }^{-}$are $(56.50-159.00 \mu \mathrm{g} / \mathrm{l}),(1.63-16.86 \mu \mathrm{g} / \mathrm{l})$ and $(28.38-97.20 \mu \mathrm{g} / \mathrm{l})$, respectively while the ranges of ortho-P and TP are $(11.00-59.40 \mu \mathrm{g} / \mathrm{l})$, and $(186.00-358.40 \mu \mathrm{g} / \mathrm{l})$, respectively. The $\mathrm{SiO}_{2}{ }^{2-}$ ranged $(5.79-12.97 \mathrm{mg} / \mathrm{l})$ and $(6.64-17.74 \mathrm{mg} / \mathrm{l})$ in the $1^{\text {st }}$ and $2^{\text {nd }}$ lake, respectively. During the seasons, there were non-significant a difference in nutrient salts levels except ortho-P and $\mathrm{SiO}_{2}{ }^{2-}$ in the $2^{\text {nd }}$ lake.

Major anions and cations ions $\left(\mathrm{HCO}_{3}{ }^{-}, \mathrm{CO}_{3}{ }^{2}, \mathrm{SO}_{4}{ }^{2-}, \mathrm{Cl}^{-}, \mathrm{Ca}^{2+}, \mathrm{Mg}^{2+}\right)$ are naturally very variable in surface waters due to local geological, climatic and geographical conditions. $\mathrm{SO}_{4}{ }^{2-}, \mathrm{Cl}^{-}, \mathrm{Ca}^{2+}$ and $\mathrm{Mg}^{2+}$ have been the same distribution trend and their concentrations in the $2^{\text {nd }}$ lake are very higher than the $1^{\text {st }}$ lake. They ranged between 281.65-372 and 563.24- 712, 51.10-68.12 and 
98.20-119.82 mg/1 and 2155.74-3418, 5073.94- 6987, 142.14-188 and 405.19$594 \mathrm{mg} / 1$ for $\mathrm{SO}_{4}^{2-}, \mathrm{Cl}^{-}, \mathrm{Ca}^{2+}$ and $\mathrm{Mg}^{2}$ in the $1^{\text {st }}$ lake and $2^{\text {nd }}$ lake, respectively. On the other hand, $\mathrm{HCO}_{3}{ }^{-}, \mathrm{CO}_{3}{ }^{2-}$ showed irregular distribution and varied between $194.56-261.12 \& 12-32 \mathrm{mg} / \mathrm{l}$ and $208.32-276.48 \& 4-18 \mathrm{mg} / \mathrm{l}$ in the $1^{\text {st }}$ lake and $2^{\text {nd }}$ lake, respectively

Heavy Metals: eight heavy metals ( $\mathrm{Fe}, \mathrm{Mn}, \mathrm{Cu}, \mathrm{Pb}, \mathrm{Ni}, \mathrm{Co}, \mathrm{Cr}$ and $\mathrm{Cd}$ ) were determined in water. The overall mean values of some heavy metals in two lakes waters are presented in Fig. (2) exception Cd is less than $0.2 \mu \mathrm{g} / \mathrm{l}$.

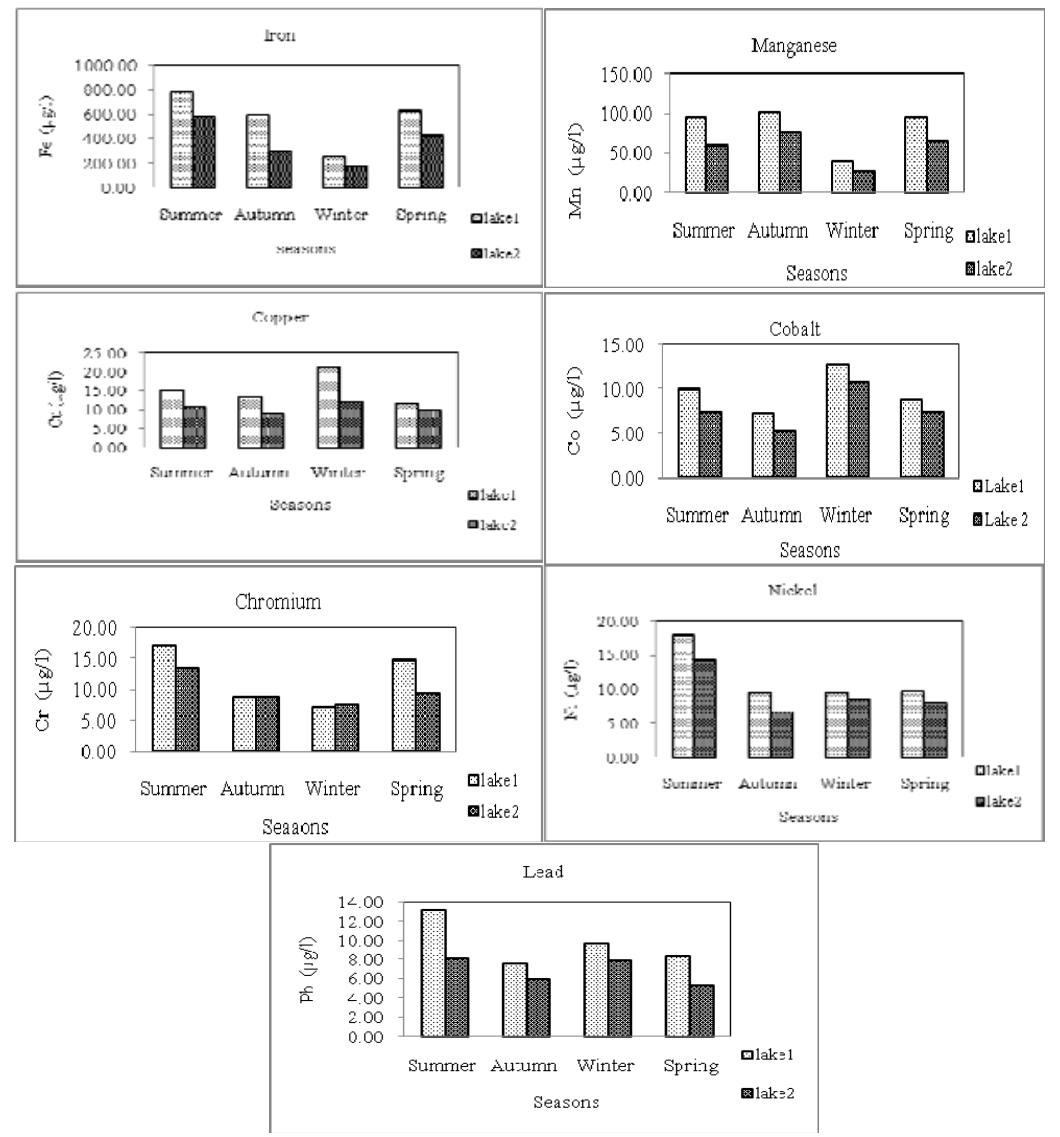

Fig. 2: Seasonal variations of the overall mean values of heavy metals in Wadi El Rayan lakes water during 2009-2010.

The results of heavy metal values showed irregular distribution in two lakes. Thus, there were non-significant differences in heavy metal levels between two lakes. Fe and $\mathrm{Mn}$ content fluctuated in wide ranges, while $\mathrm{Cu}, \mathrm{Pb}, \mathrm{Ni}, \mathrm{Co}, \mathrm{Cr}$ and $\mathrm{Cd}$ contents fluctuated in narrow ranges during the studied period. Moreover, a significant positive correlation was between heavy metals and nutrient salts.

\section{El-Wadi Drain Water}


The results of physical and chemical characteristics of El-Wadi drain water (Table 3) revealed that the effluents of El-Wadi Drain contained high values of heavy metals and nutrient Salts except $\mathrm{SiO}_{2}{ }^{2-}$ and all the remaining variables are less, compared with Wadi El Rayan lakes.

Table 3: Seasonal variations of the physico-chemical parameters and heavy metals of ElWadi Drain Water during 2009-2010.

\begin{tabular}{|c|c|c|c|c|}
\hline Variable & Summer & Autumn & Winter & Spring \\
\hline Temp. $\left({ }^{\circ} \mathrm{C}\right)$ & 29.10 & 19.80 & 16.10 & 22.10 \\
\hline $\mathrm{EC}(\mathrm{mS} / \mathrm{cm})$ & 1.64 & 1.60 & 1.49 & 1.84 \\
\hline $\mathrm{TS}(\mathrm{mg} / \mathrm{l})$ & 1346.00 & 1392.00 & 1346.00 & 1526.00 \\
\hline TDS (mg/l) & 1238.00 & 1296.00 & 1244.00 & 1412.00 \\
\hline $\mathrm{TSS}(\mathrm{mg} / \mathrm{l})$ & 108.00 & 96.00 & 102.00 & 114.00 \\
\hline $\mathrm{pH}$ & 8.18 & 7.95 & 8.19 & 8.13 \\
\hline DO (mg/l) & 6.60 & 7.20 & 7.80 & 6.80 \\
\hline $\mathrm{BOD}(\mathrm{mg} / \mathrm{l})$ & 6.20 & 6.20 & 6.80 & 4.80 \\
\hline $\mathrm{COD}(\mathrm{mg} / \mathrm{l})$ & 6.00 & 11.60 & 2.40 & 8.00 \\
\hline $\mathrm{NH}_{3}(\mu \mathrm{g} / 1)$ & 648.20 & 537.60 & 546.90 & 320.90 \\
\hline $\mathrm{NO}_{2}^{-}(\mu \mathrm{g} / 1)$ & 72.11 & 88.60 & 66.46 & 67.12 \\
\hline $\mathrm{NO}_{3}{ }^{-}(\mu \mathrm{g} / \mathrm{l})$ & 612.35 & 366.80 & 824.08 & 692.64 \\
\hline Ortho-P $(\mu \mathrm{g} / \mathrm{l})$ & 288.10 & 214.30 & 261.50 & 202.40 \\
\hline Total-P $(\mu \mathrm{g} / \mathrm{l})$ & 660.00 & 611.40 & 702.00 & 720.00 \\
\hline $\mathrm{SiO}_{2}^{2-}(\mathrm{mg} / 1)$ & 8.23 & 5.42 & 7.30 & 8.03 \\
\hline $\mathrm{CO}_{3}^{2-}(\mathrm{mg} / \mathrm{l})$ & 0.00 & 0.00 & 0.00 & 0.00 \\
\hline $\mathrm{HCO}_{3}^{-}(\mathrm{mg} / \mathrm{l})$ & 256.00 & 256.00 & 266.24 & 266.24 \\
\hline $\mathrm{Cl}^{-}(\mathrm{mg} / \mathrm{l})$ & 386.00 & 432.00 & 357.74 & 472.85 \\
\hline $\mathrm{SO}_{4}{ }^{2-}(\mathrm{mg} / \mathrm{l})$ & 236.59 & 246.00 & 195.43 & 242.94 \\
\hline $\mathrm{Ca}^{2+}(\mathrm{mg} / \mathrm{l})$ & 57.40 & 56.70 & 44.89 & 54.89 \\
\hline $\mathrm{Mg}^{2+}(\mathrm{mg} / \mathrm{l})$ & 99.60 & 87.60 & 92.77 & 98.75 \\
\hline $\mathrm{Fe}(\mu \mathrm{g} / \mathrm{l})$ & 1866.00 & 1142.00 & 2346.00 & 1186.00 \\
\hline $\operatorname{Mn}(\mu \mathrm{g} / 1)$ & 383.42 & 116.00 & 254.00 & 422.30 \\
\hline $\mathrm{Cu}(\mu \mathrm{g} / \mathrm{l})$ & 21.60 & 17.60 & 32.66 & 16.40 \\
\hline $\operatorname{Co}(\mu \mathrm{g} / 1)$ & 18.60 & 12.66 & 18.47 & 12.90 \\
\hline $\mathrm{Pb}(\mu \mathrm{g} / \mathrm{l})$ & 24.40 & 12.40 & 16.30 & 12.80 \\
\hline $\mathrm{Ni}(\mu \mathrm{g} / \mathrm{l})$ & 44.80 & 12.60 & 17.60 & 14.80 \\
\hline $\mathrm{Cr}(\mu \mathrm{g} / 1)$ & 16.35 & 7.85 & 8.13 & 8.66 \\
\hline $\mathrm{Cd}(\mu \mathrm{g} / \mathrm{l})$ & $<0.20$ & $<0.20$ & $<0.20$ & $<0.20$ \\
\hline
\end{tabular}

\section{DISCUSSION}

The lakes system of Wadi El Rayan has an exceptional importance both internationally and nationally. The water temperature is low in cold period (winter, autumn) due to frequent clouds, high humidity, high current velocity and high water level and high in hot period (summer, Spring) due to clear 
atmosphere, greater solar radiation, and low water level. Present observations are in agreement with similar ones made by Moundiotiya et al. (2004) and Lawson (2011). The $\mathrm{pH}$ values were always in the alkaline side $\mathrm{pH}$ (8.26-8.95) with small local differences without seasonal variations. This may be due to the high buffering capacity of the system. In the present study, the increase in DO concentration in winter and autumn may be due to the fall in water temperature and phytoplankton blooming (Konsowa, 2007). In general, the BOD of waste water was lower than COD, because more compound can chemically oxidized than can be biologically oxidized (Clark and Micheal, 1972), and there is an inverse relation between DO and oxygen utilization in terms of BOD and COD (Das, 2000).

The distribution of major anions and cations in lakes water were governed mainly by the rate of evaporation, the intrusion of drainage water (Abdel-Satar et al., 2010). In the $1^{\text {st }}$ lake, the concentration of major cations and anions are controlled by the intrusion of drainage water from El-Wadi Drain (Ali et al., 2007), however the concentration in the $2^{\text {nd }}$ lake are controlled by the rate of evaporation and the amount of water discharged from the $1^{\text {st }}$ lake (Abd El-karim, 2004). Generally, these are a significant differance in levels of EC, TS, TDS, TSS and major cations $\left(\mathrm{Ca}^{2+}, \mathrm{Mg}^{2+}\right)$, anions $\left(\mathrm{SO}_{4}^{2-}, \mathrm{Cl}^{-}\right)$between the two lakes, which may be due to evaporation and $\backslash$ or leaching of salts from soil as water moves deep into the desert (Saleh et al., 1988). On the other hand, the concentrations of EC, TS, TDS, TSS, major cations $\left(\mathrm{Ca}^{2+}, \mathrm{Mg}^{2+}\right)$ and anions $\left(\mathrm{SO}_{4}{ }^{2-}, \mathrm{Cl}^{-}\right)$in hot period (summer, Spring) are higher than cold period (winter, autumn).

Wadi El Rayan lakes were classified as brackishwater due to TDS values and may be salinized in a short period, particularly the $2^{\text {nd }}$ lake in the southern area. These results are in agreement and confirmed with results obtained by Sayed and Abdel-Satar (2009).

Alkalinity of carbonate and bicarbonate contants showed irregular variations during the whole period of study. These irregular variations attributed mainly to the respiration processes of aquatic microorganisms in the surface water which led to increase carbon dioxide converted to carbonate in the water environment.

The obtained results in this study revealed that nutrients $\left(\mathrm{NH}_{3}, \mathrm{NO}_{2}^{-}, \mathrm{NO}_{3}^{-}\right.$, ortho-P, TP) increase in $1^{\text {st }}$ lake, especially at the inlet of the lake due to the impact of the effluents from El-Wadi Drain which, these results are in agreement with the findings of Aboul-Ela and Khalil (1988); Saleh et al. (1988); Abd ElKarim (2004) and Sayed and Abdel-Satar (2009). However, the slightly increases of $\mathrm{SiO}_{2}{ }^{2-}$ content in the $2^{\text {nd }}$ lake, may be attributed to the $2^{\text {nd }}$ lake harbored the highest numerical density of diatoms than the $1^{\text {st }}$ lake (Sayed and Abdel-Satar, 2009). On the other hand, ammonia accounted for the major proportion of total soluble inorganic nitrogen. Nitrite showed low levels than the corresponding values of nitrate due to the fast conversion of $\mathrm{NO}_{2}^{-}$to $\mathrm{NO}_{3}{ }^{-}$ions by nitrifying bacteria (Sabae and Ali, 2004). In the most nutrient salts, the highly positive 
correlation (at $\mathrm{P}<0.05$ ) of $\mathrm{NH}_{3}$ with $\mathrm{NO}_{2}^{-}, \mathrm{NO}_{3}{ }^{-}$, ortho-P and $\mathrm{TP}$ with $\mathrm{NH}_{3}$ $\left(\mathrm{r}=0.99,0.95,0.88\right.$ and 0.77 , respectively), $\mathrm{NO}_{2}^{-}$with $\mathrm{NO}_{3}{ }^{-}$, ortho-P and $\mathrm{TP}$ $\left(\mathrm{r}=0.97,0.84\right.$ and 0.81 , respectively) and $\mathrm{NO}_{3}{ }^{-}$with ortho-P and $\mathrm{TP}(\mathrm{r}=0.83$ and 0.78 , respectively) indicates that the agriculture waste via El Wadi drain is the common source of these salts.

The concentrations of heavy metals in water of Wadi El Rayan lakes are increase at inlet the $1^{\text {st }}$ lake during the year round. Generally, the concentrations of heavy metals in the $1^{\text {st }}$ lake water are higher than the $2^{\text {nd }}$ lake which may be attributed to the effect of agricultural influx drained into the $1^{\text {st }}$ lake from ElWadi drain. These are opposed by the results of Sayed and Abdel-Satar, (2009), who reported that the concentrations of heavy metals in the $2^{\text {nd }}$ lake water are higher than the $1^{\text {st }}$ lake. On the other hand, the significant positive correlation (at $\mathrm{P}<0.05)$ between heavy metals $(\mathrm{r}=0.72,0.73,0.87,0.97 \& 0.95$ and $\mathrm{r}=0.87$, $0.60,0.99,0.93 \& 0.92$ for $\mathrm{Fe}$ and $\mathrm{Mn}$ with each of $\mathrm{Cu}, \mathrm{Co}, \mathrm{Pb}, \mathrm{Ni} \& \mathrm{Cr}$, respectively and $\mathrm{r}=0.89$ between $\mathrm{Fe}$ and $\mathrm{Mn}$ ), indicated that the association of heavy metals originates from a common source during transportation and/or depositional reactions. Fe and $\mathrm{Mn}$ are the most abundant elements in water of the two lakes whereas Cd the less one. Sayed and Abdel-Satar (2009), cited that the water of El Rayan lakes were polluted by trace metals and acts as a slow poison and adversely affects the ecology and thereby harming the flora and fauna and the natural inhabitat. Finally, these metals in trace amount may play important role in the biochemical life process of the fish (Bennett and Dooley, 1982), some as enzyme co-factor (Gatlin et al., 1982; Lovell, 1989). However, their sublethal concentrations become lethal to fish or other aquatic organisms when the duration of exposure to these metals is prolonged (Stag and Shuttleworth, 1982; Everall, 1987).

In El-Wadi Drain, the concentrations of heavy metals and nutrient salts were high except the $\mathrm{SiO}_{2}{ }^{2-}$ as compared with Wadi El Rayan Lakes. This indicated that $\mathrm{SiO}_{2}{ }^{2-}$ content of the lake not interdependent on the corresponding values in the drains but its main dependence on the biogenic silica production through diatoms. These results act in harmony with that reported by Verschuren et al. (1998) in Lake Victoria, South Africa. However, all the remaining variables are less compared with Wadi El Rayan lakes were governed mainly by the rate of evaporation, the intrusion of drainage water.

\section{CONCLUSION}

The present results revealed that the 2nd lake undergo progressive increase in salinity (TDS), especially in the last decade and the lake water may be salinized with time, leading to a great revolutionize in fauna, flora and environment of this lake. On the other hand, the discharge of pollutants via El Wadi Drain causes unfavorable impact on the lake environment. Therefore, the present study is baseline data toward future ecological study, conservation and management of the resources of these economically important lakes in Egypt. 


\section{REFERENCE}

Aboul-Ela, I. A. and M. T. Khalil (1988). Ecological studies on Wadi El-Rayan, a new lake in Egypt: Physico-chemical environment. Egypt. J. Wildlife and Natural Res., 7: 35-45.

APHA, AWW, WPCF (2005). Standard Methods for the Examination of Water and Wastewater. $21^{\text {st }}$ Edn., American Public Health Association, Washington, DC, USA.

Ali, M. H. H. (1998). Chemical and physical studies on the River Nile at Damietta branch region. M. Sci. Thesis, Fac. of Sci. Menofiya Univ.

Abd-Ellah, R. G. (1999). Physical limnology of El-Fayoum depression and their budget. Ph. D. Thesis.Fac. of Sci., South Valley Univ. (140pp).

Abd El-Karim, M. S. (2004). Ecological Studies on Periphytic Algal Communities in Wadi El-Rayian Lakes. Ph. D Thesis, Botany Department, Girls College, Ain-Shams Univ., Egypt pp: 218.

Ali, M. H. H.; A. A. Abdel-Tawab; A. M. Ali and G. G. Soliman (2007). Monitoring of Water Quality and Some Pollutants of Man-Made Lake (Wadi El-Rayan First Lake, Egypt). Egypt. J. Aquat. Biol. and fisheries, 11(3): 1235-1251.

Abdel-Satar, A. M.; M. E. Goher and M. F. Sayed (2010). Recent environmental changes in water and sediment quality of lake Qarun, Egypt. J. Fish. Aquat. Sci., 5: 56-69.

Bennett, R. O. and J. K. Dooley (1982). Cooper uptake by two sypatric species of Killi fish, Fundulus heteroclitus (L.) and F. majalis. J. Fish Biol., 21: 381-398.

Clark, B. G. and Micheal, A. U. (1972). Waste water Engineering in the water Resources and Environmental Engineering, McGrow-Hill New York.

Chaurasia, M. and G. C. Pandey (2007). Study of physico chemical characteristic of some water ponds of Ayodhya Faizabad. IJEP 27 (11): 1019-1023.

Das, A.K. (2000). Limno-Chemistry of some Andhra Pradesh Reservoirs. J. Inland. Fish. Soc. India. 32(2): 37-44. 
Everall, N.C. (1987). The effects of water hardness and Ph upon the toxicity of zinc to Brown trout, Salmo trutta. Ph.D. Thesis Trent Polytechnic Nottingham, U.K., pp: 242.

EL Bayomi, G.M. (2006). Area of Wadi El Raiyan Lakes a Geomorphological Study. J. Applied Sci. Res., 2(12):1304-1313.

El-Shabrawy, G. M. (2007). Community structure and abundance of macrobenthos in Wadi El-Rayan Lakes (El-Fayoum, Egypt). African J. Biological Science, 3(1): 113-125.

El-Shabrawy, G. M. and H. J. Dumont (2009). The Fayum Depression and Its Lakes. The Nile Monographiae Biol., 89: 463-478.

Fisher, R. A. (1970). Statistical Method for Research Workers, Eds. Oliver and Boyed, Edinburgh. Pp.140

Gatlin, D. M.; E. H. Robinson and W. E. Poe (1982). Magnesium requirements of fingerling Channel catfish. J. Nutrition, 112: 1181-1187.

IUCN (1998a). Wadi El Rayan Natural Protectorate Project. Guidelines for Overall Work Plan (1998-2001). Report 1. Prepared by G.H. Mattravers Messana.

IUCN (1998b). Wadi El Rayan Natural Protectorate Project. Guidelines for First Annual Work Plan (1998). Report 2. Prepared by G. H. Mattravers Messana.

IUCN (2000a). Wadi El Rayan Protected Area Project. Concept Outline for Second Phase. Report prepared by G.H. Mattravers Messana.

Jumbe and N. Nandini (2009). Heavy Metals Analysis and Sediment Quality Values in Urban Lakes. Am. J. Env. Sci., 5 (6): 678-687.

Kouadia, L. and J. H. Trefry (1987). Saline trace metal contamination in the Ivory Air Water Soil Pollut., 32: 145-154.

Konsowa, A. H. (2007). Phytoplankton evolution in a shallow hypertrophic saline lake. Al-Azhar J. Pharm. Sci., 32: 109-122.

Khare, K. C. and M. S. Jadhav (2008). Water Quality Assessment of Katraj Lake, Pune (Maharashtra, India): A Case Study, the $12^{\text {th }}$ World Lake Conference: 292-299. 
Lovell, R. T. (1989). Nutrition and feeding of fish. Published by Van Nostrand Reinhold New York, pp: 260.

Lawson, E. O. (2011). Physico-Chemical Parameters and Heavy Metal Contents of Water from the Mangrove Swamps of Lagos Lagoon, Lagos, Nigeria. Advances in Biol Res., 5 (1): 08-21.

Moundiotiya C.; R. Sisodia; M. Kulshreshtha and A. L. Bhatia (2004). A Case Study of the Jamwa Ramgarh Wetland With special reference to physicochemical properties of water and its Environs. J. Environ. Hydrolog., 12: $24-30$.

Mohamed, F. A. S. and N. S. Gad (2008). Environmental Pollution-Induced Biochemical Changes in Tissues of Tilapia zillii, Solea vulgaris and Mugil capito from Lake Qarun, Egypt. Global Veterinaria 2 (6): 3327-336.

Stag, R. M. and T. J. Shuttleworth (1982). The effects of copper on ionic regulation by gill of the seawater adapted flounder (Platicthys flesus, L). Comparative Physiol., 149: 83-90.

Saleh, M. A.; M. Fouda; M. A. Saleh; M. Abdel-Latif and B. Wilson (1988). Inorganic Pollution of the Wadi El Raiyan Lakes in Egypt and its Impact on their Fish and Wildlife. Archives of Env. Cont. and Toxic., 17: 391403.

S. P. S. S. (1999). Statistical Package for the Social Science, Inc. Chicago.

Sabae, S. Z. and M. H. Ali (2004). Distribution of nitrogen cycle bacteria in relation to physico-chemical conditions of a closed saline lake (Lake Qarun, Egypt). J. Egypt Acad. Environ. Dev., 5: 145-167.

Sithik, A. M.; G. Thirumaran; R. Arumugam; R. R. Kannan and P. Anantharaman (2009). Physico-Chemical Parameters of Holy Places Agnitheertham and Kothandaramar Temple; Southeast Coast of India. Am.-Eurasian J. Sci. Res., 4 (2): 108-116.

Sayed M. F. and A. M. Abdel-Satar (2009). Chemical Assessment of Wadi ElRayan Lakes-Egypt. American-Eurasian J. Agric. \& Environ. 5 (1): 53-62.

Shukla, R. and Y. K. Sharma (2009). Heavy Metal Toxicity in Environment. In: Environmental Monitoring and Management, Trivedi, A., K. Jaiswal, B. N. Pandey and S.P. Trivedi (Eds.). Alfa Publications, ISBN-978-81907843-8-2, pp: 137-162. 
Usharani, K.; K. Umarani; P. M. Ayyasamy and K. Shanthi (2010). Physicochemical and bacteriological characteristics of Noyyal river and ground water quality of Perur, India. J. Appl. Sci. Environ. Manage, 14(2): 29-35.

United States Environmental Protection Agency (USEPA) (2009). National Recommended Water Quality Criteria for Priority Pollutants. URL: http://www.epa.gov/ost/criteria/wqctable/

Verschuren, D.; Edgington, D. N.; Kling, H. J. and Johnson, T. C. (1998). Silica depletion in Lake Victoria: Sedimentary signals at offshore stations. J. Great Lakes Res. 24 (1): 118-130.

Welch, P. S. (1952). Limnology. $2^{\text {nd }}$ Ed. Mc. Graw Hill Book Comp, New York, U.S. A.

Waller, W. M. and Duncan, D. B. (1969). A bays role for symmetric multiple composition problem. J. Am. State. Assoc. J. 64: 1484.

Wetzel, R. G. (1975). Limnology. W. B. Saunders Co., Philadelphia, London, and Toronto. xii +743 . 